\title{
Zur Bedeutung des fremdsprachlichen Schreibens. Einige Überlegungen aus der Theorie und Praxis des DaF-Unterrichts auf der Fortgeschrittenenstufe
}

\author{
About the Meaning of Writing \\ in a Foreign Language. Reflections in the Context \\ of the Theory and Practice of Teaching German \\ as a Foreign Language to Advanced Students
}

ABSTRACT. The article aims at analyzing the approach to writing skills in selected foreign language teaching methodologies and at characterizing the function of writing from the perspective of the theory and practice of teaching German as a foreign language.

KEYWORDS: creative writing, foreign language learner, language teaching methods, process of writing, German as a foreign language, writing skills.

\section{VORBEMERKUNG}

Während man noch vor einigen Jahrzehnten Schreiben als eine eher vernachlässigte Fertigkeit zu bezeichnen pflegte, kann heutzutage im Hinblick auf die technischen der zwischenmenschlichen Kommunikation verpflichteten Errungenschaften eher von seiner Renaissance gesprochen werden. Wie Starke, Zuchewicz (2003: 9) mit Recht betonen, ist das geschriebene Wort in der digitalen Welt sehr stark im Kommen, weil die übliche Gesprächssitua- 
tion, in der sich zwei Partner gegenüber stehen und miteinander kommunizieren, im Alltag und am Arbeitsplatz eben durch das Internet immer seltener wird, wobei an dieser Stelle unbedingt zu bemerken ist, dass der durchschnittliche Internet-Benutzer so schreibt, wie er spricht, was sich wiederum in der Qualität der E-Mail-Produkte deutlich widerspiegelt. Es verwundert daher nicht, dass viele Studierende - darunter auch Philologen - beim Schreiben studienrelevanter Textsorten oft in Schwierigkeiten geraten oder sogar daran scheitern, wenn sie weder in der Schule noch in schreibintensiven Studienfächern in die Schreibfertigkeit nicht systematisch eingeführt werden.

Im präsentierten Beitrag wird daher der Versuch unternommen, theoretische Überlegungen zur Rolle des fremdsprachlichen Schreibens den Studentenmeinungen zu demselben Thema gegenüberzustellen, um schließlich einen Vergleich zwischen den beiden Ebenen zu wagen. Da jedoch ein enger Zusammenhang zwischen ihnen besteht, hat man sich absichtlich dazu entschlossen, jene nicht separat zu behandeln, sondern die Ergebnisse der durchgeführten Befragung jeweils mit theoretischen Erwägungen zu untermauern.

\section{EINSTELLUNG ZUR FERTIGKEIT SCHREIBEN IN DEN AUSGEWÄHLTEN METHODEN DES FREMDSPRACHENUNTERRICHTS}

In der Geschichte des Fremdsprachenunterrichts unterlag offensichtlich nicht nur der Stellenwert der Muttersprache sowie der fremdsprachlichen Grammatik Veränderungen verschiedenster Art. ${ }^{1}$ Auch die Schreibfertigkeit wurde im Laufe der Jahrhunderte unterschiedlich aufgefasst - sowohl ein Schattendasein führend als auch im Vordergrund stehend - was sich gerade am Beispiel der ausgewählten Methoden des Fremdsprachenunterrichts deutlich illustrieren lässt.

In der auf dem Unterricht in klassischen Sprachen wie Griechisch oder Latein aufbauenden Grammatik-Übersetzungs-Methode, die auf die Vermittlung von Sprachwissen gerichtet ist, um vorgegebene grammatisch korrekte Sätze verstehen, bilden und übersetzen zu lernen (Skowronek 2008: 398), hat die Schriftlichkeit ihren unbestrittenen Vorrang vor anderen Fertigkeiten. Jene Blütezeit des Geschriebenen endet jedoch mit der des Öfteren als Anti-Grammatik-Methode bezeichneten direkten Methode, in der das mühsam erstrittene Primat des Mündlichen gilt und Schreiben als vierte der fremdsprachlichen Fertigkeiten ganz in den Hintergrund rückt, weil sie als

${ }^{1}$ Ausführlich dazu Pfeiffer (2001). 
sekundäre, der gesprochenen Sprache unterworfene, Tätigkeit abgewertet wird (Krumm 2000: 7).

Auch mit dem Einzug der audiolingualen Methode in den Fremdsprachenunterricht ändert sich die Position der Fertigkeit Schreiben nicht erheblich, denn, wie Kuligowska (2008: 49) akzentuiert, leidet auch jener am Schreibentzug. In den Vordergrund wird die Kommunikationsfähigkeit gestellt, die sich jedoch auf die gesprochene Sprache beschränkt. Daraus ergeben sich auch die Konsequenzen für die Reihenfolge der Aneignung der einzelnen Fertigkeiten: Hörverstehen - Sprechen - Leseverstehen - Schreiben, was für den gegebenen Text wiederum Folgendes bedeutet: Erst hören, danach mündlich einüben, dann lesen und erst zu einem späteren Zeitpunkt schreiben (z.B. schriftgestützte Reproduktion oder schriftliche Übungen), was jedoch nicht selten im Sinne einer längeren schriftfreien Anfangsphase beim Fremdsprachenlernen verstanden wird (Storch 1999: 249). Der Unterricht verläuft demnach, wie Skowronek (2000: 398) bemerkt, ohne kreativen Beitrag des Lerners, denn erreicht wird nur fehlerfreies, auswendig gelerntes Nachsprechen der vom Lehrer vorgegebenen Beispiele anstatt einer spontanen, natürlichen, gesellschaftlich effektiven Kommunikation.

Mit der Ende der 1970er Jahre eingetretenen kommunikativ-pragmatischen Wende versucht man zwar grundsätzlich die vier Sprachfertigkeiten als gleichwertig einzustufen, aber in der Praxis kommt der Fertigkeit Schreiben eine untergeordnete Rolle $\mathrm{zu}$, denn eine Beschäftigung mit dem zeitaufwendigen Prozess des Schreibens könnte die Konzentration auf die Kommunikationsfähigkeit behindern (Krischer 2002: 384). Nach Roche (2005: 200-201) wurde Schreiben im Rahmen der kommunikativen Didaktik somit lange als nicht kommunikativ abgetan, bis man endlich zur Einsicht kam, wie viele Schreibproduktionsprozesse beim Schreiben verlaufen. Daher wird heutzutage darauf verwiesen, dass dabei das innere Sprechen der eigentlichen Verschriftlichung vorangeht und es daher mehr sprachliche Aktivitäten verlangt als die Zeilen an der Oberfläche vermuten lassen.

Die Erkenntnis darüber, dass man für ein erfolgreiches Fremdsprachenerlernen alle Sprachfertigkeiten gleichrangig behandeln sollte, rückt also allmählich in den Vordergrund, wobei auch berücksichtigt werden muss, dass der Schreibfertigkeitserwerb der komplexeste und deswegen auch der anspruchsvollste ist. Beim Schreiben bieten sich jedoch Möglichkeiten, mit denen man seine Aneignung entwickeln kann. Fremdsprachenlernenden sollte daher ermöglicht werden, beim Schreiben ihre Kreativität zum Ausdruck zu bringen, womit ihre Bereitschaft angeregt wird, auch in der Fremdsprache Schreiben als ein Kommunikationsmedium bewusst anzuwenden, das auf der Sprachnormenbeherrschung und dem Verhältnis zu sich selbst wie auch zur Umwelt, in der es entsteht, beruht (Kosevski Puljić 2009: 92). 
Kast (1999: 22) weist darauf hin, dass im kommunikativen Ansatz Sprache und somit auch Schreiben als soziales Handeln aufgefasst wird. Es wird geschrieben, um Produkte (d.h. Informationen) zu erhalten. Man schreibt also beispielsweise, um sich zu beschweren (Geschäftsbriefe), um nichts zu vergessen (Einkaufszettel), um einen Leistungsnachweis zu erhalten (Tests) usw. Zwar ist jener mitteilungs- und produktorientierte Aspekt des Schreibens von hoher Relevanz, aber das Schreiben hat noch andere Funktionen zu erfüllen, auf die im weiteren Teil des Beitrags eingegangen wird.

Eigentlich kann man aufgrund der oben dargestellten Analyse zum Stellenwert der Schreibfertigkeit in den einzelnen Methoden des Fremdsprachenunterrichts auf keinen Fall behaupten, dass Schreiben immer vernachlässigt wurde und somit eine geringfügige Rolle spielte. Bereits Krumm (1989: 5) hat mit Recht hervorgehoben, dass Lehrbücher und vor allem die Arbeitsbücher zu den Lehrwerken zahlreiche Schreibaufgaben enthalten. Tatsächlich wurde und wird im Unterricht immerzu geschrieben; vom Abschreiben an der Wandtafel über Diktate, Nacherzählungen bis zu Grammatikübungen verschiedenster Art. Allerdings sind leider kaum Anzeichen einer systematischen Schreibentwicklung zu finden, denn das Schreiben läuft im Unterricht vielmehr einfach mit, je nach Lehrer mit größerer oder geringerer Aufmerksamkeit. Erst in den letzten Jahrzehnten des vergangenen Jahrhunderts hat - ausgehend vom muttersprachlichen Deutschunterricht - hier ein Wandel eingesetzt, der in verschiedenen Entwicklungen begründet ist. Zu erwähnen sind an dieser Stelle:

- eine stärkere Auseinandersetzung der Sprachwissenschaft mit den Unterschieden zwischen gesprochener und geschriebener Sprache (so liefert z. B. die Duden-Grammatik erstmals in der Neuauflage von 1984 ein eigenes Kapitel zu diesen Unterschieden),

- Untersuchungen der Kultur- und Literaturwissenschaft, wie weit bestimmte Textformen kulturgeprägt sind, so dass Schreibenlernen in der Fremdsprache auch das Erlernen neuer kultureller Ausdrucksmöglichkeiten erfordert,

- eine genauere Beschäftigung der Lernpsychologie mit dem Prozess des Schreibens und die Entwicklung von Schreibmodellen, die Grundlage für eine gezielte Schreibförderung sein können,

- Veränderungen von Kommunikationsbedürfnissen: Das durch Telefon und Fernsehen verdrängte Schreiben wird durch Computer und Telefax wieder zu einem modernen Kommunikationsmedium.

Auch Zuchewicz (2001: 14) teilt Krumms Standpunkt, wenn er darauf hinweist, dass im und für den Unterricht viel geschrieben wurde, jedoch in erster Linie mit dem Ziel, bestimmte Strukturmuster und (umgangs)sprachliche Wendungen für den mündlichen Sprachgebrauch $\mathrm{zu}$ automatisieren, 
was in der Schreibforschung als Mündlichkeit im Gewande der Schriftlichkeit bezeichnet wird.

Die bei Krumm (1989: 5) oben genannten Tendenzen haben sicherlich einen nicht geringen Beitrag zur Aufwertung des Schreibens geleistet, was wiederum wesentliche Veränderungen in seiner Auffassung im modernen Fremdsprachenunterricht nach sich zieht, zumal es zwar als ein durchaus komplexer Prozess zu betrachten ist, dessen Förderung hohe Anforderungen an Lehrende und Lernende stellt, aber im Hinblick auf seine mannigfachen Funktionen, die auch im Beitrag thematisiert werden, aus dem (Schul)Alltag zweifelsohne nicht wegzudenken ist.

\section{DIE ROLLE DER SCHREIBFERTIGKEIT VOR DEM HINTERGRUND DER THEORIE UND PRAXIS DES FREMDSPRACHENUNTERRICHTS}

Die Verfasserin des Beitrags wurde u.a. von den oben präsentierten Überlegungen zum Stellenwert des Schreibens in der Geschichte des Fremdsprachenunterrichts zum Durchführen einer Pilotstudie verleitet, an der insgesamt 20 Germanistikstudenten des dreijährigen Bachelor-Studienganges an der Adam-Mickiewicz-Universität in Poznań (fünf Personen im ersten Studienjahr und fünfzehn Personen im dritten Studienjahr) im Wintersemester 2011/2012 teilgenommen haben. ${ }^{2}$

Das Hauptanliegen der qualitativ ausgerichteten und durch schriftliche Befragung, teilnehmende Beobachtung sowie Dokumentenanalyse begleiteten Forschung lag darin, zu beweisen, dass es durchaus sinnvoll und begründet ist, kreatives Schreiben auf der Fortgeschrittenenstufe des Fremdsprachenunterrichts $\mathrm{zu}$ fördern. Daraus ergaben sich selbstverständlich weitere für das Forschungsvorhaben relevante Fragen, u.a. folgende: Kann kreatives Schreiben einen Beitrag dazu leisten, Schreibblockaden bei Lernern abzubauen? Muss Schreiben immer, wie die gängige Studentenmeinung lautet, für schwierig, langwierig, zeit- und arbeitsaufwendig gehalten werden oder lässt sich jene Einstellung durch kreatives Schreiben ändern? Übt kreatives Schreiben einen Einfluss auf die Erhöhung der Schreibkompetenz und somit der interkulturellen kommunikativen Kompetenz aus? Wie sollte kreatives Schreiben auf der Fortgeschrittenenstufe (u.a. in deutsch-polnischen Tandems $)^{3}$ gestaltet werden, damit Lerner davon optimal profitieren

\footnotetext{
${ }^{2}$ Im ersten Studienjahr wurde die Untersuchung in eine zusätzlich veranstaltete Sitzung und im dritten Studienjahr in ein regelmäßig stattfindendes Seminar integriert.

${ }^{3}$ Besonderer Dank gilt an dieser Stelle Frau Dr. Sonja Vandermeeren von der ChristianAlbrechts-Universität in Kiel und ihren fünf DaF-Studierenden sowie Herrn Dr. Matthias
} 
können? Ist Schreiben gelungener Texte auf kreative Weise überhaupt lernbar?

Nachdem den Studierenden die Hauptziele sowie der Ablaufplan der Pilotstudie präsentiert worden waren, wurden sie nach ihren Assoziationen zu fremdsprachlichem Schreiben und dessen Funktionen befragt. Einige haben darauf hingewiesen, dass es eine recht gute Gelegenheit ist, eigene Gedanken zu Papier zu bringen, sie zu versprachlichen oder sogar zu präzisieren, denn gerade beim Schreiben ist es durchaus möglich, sich Zeit zu nehmen, um einer Sache auf den Grund zu gehen bzw. viele Einzelheiten zu berücksichtigen. In diesem Kontext ist auf Werner Ingendahls Buch (1972) Aufsatzerziehung als Hilfe zur Emanzipation zu verweisen, in dem der Verfasser dem Schreiben keine kommunikative, sondern eine heuristische Bedeutung beigemessen hat, weil man dadurch zu eigenen Erkenntnissen gelangen und die personale Entwicklung von Lernern fördern kann. Seine Konzeption gründete auf dem russischen Psychologen Lew Semjonowitsch Wygotski, der die geschriebene Sprache nicht primär für kommunikativ hielt, da auf einzelne Sätze oder Textsegmente keine Antworten folgen, die wiederum den folgenden Text beeinflussen könnten. Schreiben ist für ihn somit eine monologische Form (Huneke, Steinig 2004: 111). Auch Kästner, Mummert (2000: 229-230) knüpfen an jenen wichtigen Aspekt des Schreibens an, wenn sie hervorheben, dass sich Bewusstseinsprozesse immer dann vollziehen, sobald exemplarische Erlebnisse mit und in der fremden Kultur in Sprache transformiert werden, wobei man sich ihrer besonders im Auf- und Beschreiben von Erinnerungen/ Erfahrungen vergegenwärtigt, wodurch sie auch verständlicher werden. Die Schreibenden machen sich einen Begriff von dem, was ihnen als Gefühl oder als loser Gedanke vorschwebt. Im besten Falle begreifen sie sich selbst etwas besser und damit auch ihre individuelle Sicht auf die fremde/eigene Kultur, was wiederum zu Irritationen und somit zu (Denk-)Problemen, zu weiteren Fragen oder eben zu neuem Wissen führen kann. Jenes Sich-Wundern über sich selbst, über das Neue/ Fremde über den/das Gegenüber oder über Das-bisher-so-nicht-Betrachtete erlangt erst in (Schrift-)Sprache übersetzt eine gewisse Konsistenz, denn es wird sozusagen dingfest gemacht.

Der Schreibprozess ermöglicht es, das eigene Individuum, die umgebende Wirklichkeit zu reflektieren, was jedoch nicht immer sofort zu einer eindeutigen und problemlos gewonnenen Erkenntnis führen muss. Schreiben wird demnach als ein Vorgang betrachtet, dessen Ergebnisse nicht unbe-

Springer von der Ludwig-Maximilians-Universität in München und seinen neun DaF-Studierenden, dank deren Engagement der das Schreiben in deutsch-polnischen Tandems betreffende Teil der Pilotstudie zu einem erfolgreichen Unternehmen hat werden können. 
dingt ad hoc zu erwarten sind, denn Gedanken nehmen auf Papier nur allmählich Gestalt an, um auch jederzeit einer Verifizierung bzw. Falsifizierung unterzogen werden zu können. Daher hebt auch Kast (1999: 23) hervor, Gedanken, Ideen, Einsichten und Erkenntnisse seien häufig nicht von Anfang an voll entwickelt da, sondern sie entstünden und reiften während des Schreibens. Dabei spielen zwei wichtige Merkmale des Schreibens eine wichtige Rolle: die Vergegenständlichung der Gedanken und Gefühle (das zu Papier oder auf den Computer Gebrachte sieht man vor sich, man spürt Nähe - das genau wollte man ausdrücken - und Distanz - es ist noch nicht genau das, was man sagen wollte) und die Verlangsamung der Abläufe (Gedanken sind schnell. Beim Schreiben gewinnt man Zeit, man kann ordnen, Gedankenblitze fixieren).

Unter den Befragten ließen sich noch Stimmen vernehmen, die behaupteten, Schreiben in der Fremdsprache sei eine reine und dazu noch besonders zeit- und arbeitsaufwendige Qual. Sicherlich steht dies in engem Zusammenhang damit, wie Fix (2008: 226-227) bemerkt, dass Schreiben und Denken eng miteinander verbunden seien und je mehr etwas sprachlich explizit auf den Punkt gebracht werden müsse, umso mehr Widersprüche des bereits Formulierten würden deutlich, die in der Folge beseitigt werden müssten, was wiederum zu neuen Widersprüchen führe. ${ }^{4}$ Offensichtlich bemängelten also die Studierenden eine ausreichend entwickelte Schreibkompetenz bei sich, was wiederum, wie eingangs formuliert wurde, aus dem Schreiben einen mühsamen und nicht immer von Erfolgen gekrönten Prozess macht. Starke, Zuchewicz (2003: 19) heben mit Recht hervor, dass mit der Beherrschung der Schreibmotorik und der Schriftzeichen der jeweiligen Sprache zwar Grundlagen für das Schreibkönnen geschaffen werden, doch es gilt damit keinesfalls als erworben. Sie vergleichen das Schreiben mit einem gängigen Musikinstrument, in dessen Besitz praktisch jeder kommen kann. Nicht allen gelingt es aber, das Instrument gleich gut zu beherrschen. Während sich manche schon mit einfachen Tönen zufriedengeben, versuchen andere wiederum, nach Noten zu spielen. Dabei lassen sie immer mehr die eigene Kreativität zu Worte kommen, bis sie eines Tages selber schöpferisch wirken. Der Weg dahin führt jedoch über mehrere Stufen, auf denen man systematisch übt, die bereits erworbenen Fähigkeiten mühselig wiederholt, vervollkommnet und ständig neue Herausforderungen auf sich nimmt, was weder allein durch Talent noch durch überdurchschnittliche Intelligenz kompensiert werden kann.

Während der Diskussion wurde noch eine weitere Funktion des Schreibens erwähnt: Schreiben als Medium der Kommunikation. In der vom Inter-

${ }^{4}$ Man spricht demnach von der sog. dialektischen Barriere (vgl. dazu Dörner 1979). 
net dominierten Welt wird heutzutage besonders viel geschrieben, denn die meist indirekte Kommunikation verläuft vorwiegend auf der Ebene von E-Mails und SMS. In Bezug auf das Fremdsprachenlernen und -lehren eröffnet das Internet den Lernern interessante Möglichkeiten, mit Benutzern der Zielsprache Kontakt aufzunehmen, um auf diese Weise u.a. eigene Sprachkenntnisse einer Überprüfung zu unterziehen bzw. sie zu erweitern. Auf diese Rolle des Schreibens weist Wolff (1997: 53) hin, der jene in den von Informationstechnologen entwickelten Kommunikationsmodi begründet sieht, die eben auf der Übertragung geschriebener Sprache basieren. Daraus ergibt sich auch eine Konsequenz für die Nutzer - wenn man sich dieser Kommunikationskanäle bedienen will, muss man schreiben können, zumal Menschen, wenn es um berufliche Qualifikationen geht, vor allem auf der Grundlage ihrer Fähigkeit angemessene geschriebene Texte produzieren zu können (Briefe, Lebensläufe etc.), eingeschätzt werden.

Für eine recht große Gruppe von Probanden bedeutete fremdsprachliches Schreiben auch ein wichtiges Hilfsmittel im Lernprozess. So argumentierten sie beispielsweise, dass sie im Studium zahlreiche Notizen anfertigen, lexikalische Aufgaben bewältigen, Grammatikübungen machen (müssen) usw. Schreiben fungiert also ihrer Meinung nach nicht ausschließlich als Ziel an sich, sondern es dient als Mittel bzw. Instrument, um ein (Lern-)Ziel zu erreichen. Schreiben als Mittel der Fremdsprachenaneignung erhöht demnach den Einprägungseffekt, ermöglicht zeitgleich individuelle produktive Sprachtätigkeiten aller Lerner im Unterricht und bietet daher günstige Möglichkeiten für ein aufgabendifferenziertes Arbeiten im Hinblick auf den Umfang der Schreibhandlungen, die Art der Schreibaufgabe, die verfügbare Zeit sowie die Selbständigkeit der Lerner beim Lösen der Schreibaufgabe (Borgwardt et al. 1993: 141). Es ist nicht zu übersehen, dass im Allgemeinen geschriebene oder zu schreibende Texte beim Lerner einen höheren Einpräge- und Behaltenseffekt als gesprochene Texte bewirken, was wiederum mit dem langsameren Verlauf, dem größeren Aufwand und der schwer zu erlangenden Geläufigkeit von Schreibhandlungen zu begründen ist (Bohn 1996: 111). Schreiben als Zieltätigkeit liegt dagegen immer dann vor, wenn Lerner Texte in der geschriebenen Sprache, nicht nur zu realen Schreibanlässen (z.B. persönliche Stellungnahme zum Thema), sondern auch zu simulierten Schreibanlässen (klasseninterner Leserbrief, Einkaufszettel für ein Rollenspiel) produzieren (Storch 1999: 249).

Zweifelsohne dient Schreiben auch der Leistungskontrolle, worauf auch einige Studierende eingegangen sind, was Iluk (1997: 12) mit dem inzwischen über Jahrzehnte nicht begründeten Standpunkt erklärt, nach dem Schreiben a priori zum Einprägen von Lexik, Grammatik und als Mittel der vorher erwähnten Kontrolle prozeduralen und deklarativen Wissens fun- 
giert. Bereits Fritzsche (1984: 295) betonte, dass kein anderes Handlungsfeld des Deutschunterrichts so vom Beurteilen, Kontrollieren, Zensieren bestimmt und verdorben wird wie die schriftliche Kommunikation. Daran scheint sich nicht viel geändert zu haben, denn

In der Regel wird dem Schreiben eine einzige Aufgabe zugeordnet, nämlich irgendwelche Inhalte so korrekt wie möglich aus dem Kopf auf das Papier zu befördern. Dabei lautet die Devise: Erst klar denken, dann klar formulieren (Pyerin 2001: 13).

An dieser Stelle könnte man auch nach Ortner (1998: 46) eine nicht gerade leicht $\mathrm{zu}$ beantwortende und sicherlich zu regen Diskussionen verleitende Frage stellen: Wie soll der Schüler Wissen schaffend schreiben, wenn er immer nur Wissen wiedergebend geschrieben hat? Vielleicht sollte man hier, Ortners Worte ergänzend, noch eine weitere Frage wagen, deren Beantwortung einer soliden empirischen Untermauerung bedarf: Kann man in kreativem Schreiben die Chance sehen, produktives Schreiben zu fördern?

Schreiben ermöglicht es offensichtlich auch, Gefühle zum Ausdruck zu bringen, sie zu verarbeiten, wobei, wie einer der Studenten betont hat, in erster Linie die Möglichkeit einer zeitversetzten und nicht einer für den Textproduzenten anspruchsvollen, weil unter Zeitdruck stehenden, zeitgleichen Kommunikation für den Schreibenden von besonderem Vorteil zu sein scheint, zumal man im Umgang mit den eigenen Emotionen immer vorsichtig sein sollte.

Der Schreibprozess verleitet somit zu einer vertieften Reflexion:

Im Schreibakt besteht die Möglichkeit, sich über die Dinge und über sich selbst klar zu werden. Man verändert sich dadurch selbst. Auf diesem Wege hinterläßt das Schreiben nicht nur auf dem Papier Spuren, sondern auch in Seele und Geist (Ngatscha 2004: 427).

Bünting, Bitterlich, Pospiech (2009: 11-12) unterscheiden dabei neben der bereits erwähnten expressiven Funktion geschriebener Texte, die man beispielsweise an Wänden, auf Schul- und Hörsaalbänken lesen kann, zwischen ihrer deskriptiven, informativen und somit in der Hochschule vorherrschenden Funktion, ihrer direktiven, instruktiven Rolle und schließlich ihrer appellativen Bedeutung, welche die Aufmerksamkeit der Angesprochenen und Leser wecken soll.

Anhand der oben präsentierten theoretisch und empirisch fundierten Überlegungen zum fremdsprachlichen Schreiben lässt sich sein mehrdimensionaler Charakter bestätigen, wobei sicherlich die Tatsache, dass sich die Befragten seiner mannigfachen Funktionen bewusst sind und offensichtlich jene zu schätzen wissen, obschon sie allerdings einen relevanten Aspekt von 
Schreiben übersehen haben, besonders erfreulich ist. Durch fremdsprachliches Schreiben kann man einen Einblick in kulturspezifische Textsorten gewinnen, die wiederum in engem Zusammenhang mit bestimmten Erwartungen an Schreiber stehen, weil sie sehr stark in die jeweilige Zielsprachenkultur eingebettet sind und diese somit widerspiegeln.

\section{ABSCHLIESSENDE BEMERKUNGEN}

Wie oben gezeigt werden konnte, weist das fremdsprachliche Schreiben eine breite Palette von Möglichkeiten im (Schul-)Alltag auf, die von der Entfaltung des eigenen Ich über die Bewältigung studienrelevanter Aufgaben und Wissensbildung bis zur Verständigung mit der Umgebung reichen und sicherlich noch um weitere im Beitrag nicht genannte Funktionen erweitert werden könnten. Daher sollte die fremdsprachliche Schreibfertigkeit zweifelsohne als ein durchaus komplexer Prozess gefördert werden, indem man die Lerner in ihren Experimenten mit dem geschriebenen Wort durch die mannigfachen das Planen, Formulieren und Überarbeiten erleichternden Aktivitäten unterstützt, wozu gerade auch kreatives Schreiben Lehrenden und Lernenden interessante und konstruktive Gelegenheiten eröffnet.

\section{LITERATURVERZEICHNIS}

Bohn, R., 1996. Schreiben. In: Henrici, G., Riemer, C. (Hrsg.). Einführung in die Didaktik des Unterrichts Deutsch als Fremdsprache mit Videobeispielen. Baltmannsweiler: Schneider Verlag Hohengehren, 103-127.

Borgwardt, U., Enter, H., Fretwurst, P., Walz, D., 1993. Kompendium Fremdsprachenunterricht. Ismaning: Max Hueber Verlag.

Bünting, K.-D., Bitterlich, A., Pospiech, U., 2009. Schreiben im Studium: mit Erfolg. Ein Leitfaden. Berlin: Cornelsen Verlag Scriptor GmbH \& Co. KG.

Dörner, D., 1979. Problemlösen als Informationsverarbeitung. Stuttgart: Kohlhammer.

Duden Bd. 4 1984. Grammatik der deutschen Gegenwartssprache. Mannheim: Dudenverlag.

Fix, M., 2008. Texte schreiben. Schreibprozesse im Deutschunterricht. Padeborn: Verlag Ferdinand Schöningh GmbH \& Co. KG.

Fritzsche, J., 1984. Schriftliche Kommunikation. In: Hopster, N. (Hrsg.). Handbuch "Deutsch“ für Schule und Hochschule. Padeborn-München-Wien: Ferdinand Schöningh, 281-306.

Huneke, W., Steinig, H.-W., 2004. Sprachdidaktik Deutsch. Berlin: Erich Schmidt Verlag.

Iluk, J., 1997. Curriculare Entscheidungen zur Entwicklung der Schreibfertigkeit im Fremdsprachenunterricht. In: Iluk, J. (Hrsg.). Probleme der Schreibentwicklung im Fremdsprachenunterricht. Katowice: Wydawnictwo Uniwersytetu Śląskiego, 11-31.

Ingendahl, W., 1972. Aufsatzerziehung als Hilfe zur Emanzipation. Didaktik und Methodik schriftlicher Sprachgestaltung. Düsseldorf: Pädagogischer Verlag Schwann.

Kast, B., 1999. Fertigkeit Schreiben. Berlin München Wien: Langenscheidt. 
Kästner, U., Mummert, I., 2000. Schreibbegegnungen - Begegnungsschreiben. Ein Projekt mit polnischen und deutschen Studierenden zur interkulturellen Verständigung durch Freies Schreiben. In: Krumm, H.-J. (Hrsg.). Erfahrungen beim Schreiben in der Fremdsprache Deutsch. Innsbruck: Studien Verlag GmbH, 225-268.

Kosevski Puljić, B., 2009. Der Erwerb der Schreibkompetenz im Deutsch als Fremdsprache zwischen Konzepten und Modellen. In: Glottodidactica XXXV, 91-101.

Krischer, B., 2002. Schreiben - aber wie? In: Info DaF 5, 383-408.

Krumm, H.-J., 1989. Thema „Schreiben“. In: Fremdsprache Deutsch 1, 5-8.

Krumm, H.-J., 2000. Schreibforschung und Schreibförderung im Deutsch als FremdspracheUnterricht. In: Krumm, H.-J. (Hrsg.). Erfahrungen beim Schreiben in der Fremdsprache Deutsch. Innsbruck: Studien Verlag, 7-16.

Kuligowska, K., 2008. Kreatives Schreiben - eine gute Möglichkeit Schüler zum Schreiben in der Fremdsprache anzuregen. In: Studia Germanica Posnaniensia XXXI, 147-155.

Ngatscha, A., 2004. Was kann Aktionsforschung zur Praxis des Fremdsprachenunterrichts Deutsch beitragen? In: Info DaF 4, 422-430.

Ortner, H., 1998. Das kreative Schreiben - halbierte Kreativität und halbierte Schriftlichkeit? In: Zeitschrift für den Deutschunterricht in Wissenschaft und Schule 4, 37-52.

Pfeiffer, W., 2001. Nauka języków obcych. Od praktyki do praktyki. Poznań: Wagros.

Pyerin, B., 2001. Kreatives wissenschaftliches Schreiben. Tipps und Tricks gegen Schreibblockaden. Weinheim München: Juventa.

Roche, J., 2005. Fremdsprachenerwerb. Fremdsprachendidaktik. Tübingen: Narr Francke Attempto Verlag $\mathrm{GmbH}+\mathrm{Co} . \mathrm{KG}$.

Skowronek, B., 2008. Fremdsprachenunterricht als Vorbereitung auf die gesellschaftlich effektive Kommunikation. In: Mikołajczyk, B., Kotin, M. (Hrsg.). Terra grammatica. Ideen - Methoden - Modelle. Festschrift für Józef Darski zum 65. Geburtstag. Frankfurt am Main: Peter Lang $\mathrm{GmbH}, 393-402$.

Starke, G., Zuchewicz, T., 2003. Wissenschaftliches Schreiben im Studium von Deutsch als Fremdsprache. Frankfurt am Main: Peter Lang GmbH.

Storch, G., 1999. Deutsch als Fremdsprache. Eine Didaktik. München: Wilhelm Fink Verlag.

Wolff, D., 1997. Der Computer als Hilfsmittel und Werkzeug bei der Förderung muttersprachlicher und zweitsprachlicher Schreibkompetenz. In: Iluk, J. (Hrsg.). Probleme der Schreibentwicklung im Fremdsprachenunterricht. Katowice: Wydawnictwo Uniwersytetu Śląskiego, 51-73.

Zuchewicz, T., 2001. Befähigung zum wissenschaftlichen Schreiben in der Fremdsprache Deutsch. In: Deutsch als Fremdsprache 1, 14-19. 